\title{
Chronic Pancreatitis and Systemic Lupus Erythematosus: An Uncommon Association
}

\author{
Silvia C. Gutierrez ${ }^{\mathrm{a}} \quad$ Analia Veronica Pasqua ${ }^{\mathrm{a}}$ \\ Horacio Casas ${ }^{b}$ Maria Blanca Cremaschi ${ }^{c}$ \\ Marcela Liliana Valenzuela ${ }^{d} \quad$ Ariel Adalberto Cubilla $^{d}$ \\ Adriana Garcia ${ }^{e}$
}

a Service of Gastroenterology, bSection of Immunology and 'Service of Internal Medicine, Department of Medicine, Hospital Posadas, Buenos Aires, Department of Imaging, dHospital Posadas and eHospital Britanico, Buenos Aires, Argentina

\section{Key Words}

Chronic pancreatitis - Pancreas · Systemic lupus erythematosus

\begin{abstract}
The association between systemic lupus erythematosus (SLE) and chronic pancreatitis $(C P)$ is extremely rare. Up to now, only six cases have been reported. We report the case of a young woman who presented her first episode of abdominal pain and hyperamylasemia at the onset of SLE and developed chronic calcifying pancreatitis after a two year period.
\end{abstract}

\section{Case Report}

A 26-year-old white woman was admitted to our hospital in August, 2003. At that time she presented with fever, abdominal pain, diminished bowel sounds, rash, psychotic disturbances, anemia, leukopenia, raised liver associated enzymes, hyperamylasemia, low complement level and some positive antibodies (antinuclear - titer 1/1,600 - anti-DNA, anti-Sm, anti-Ro). Anti-smooth muscle, anti-liverkidney microsomal and anti-mytochondrial antibodies were negative. Viral hepatitis $(A, B, C)$ and human immunodeficiency virus (HIV) infections were ruled out. An abdominal ultrasound (US) showed a diffuse increase in echogenicity and enlargement of the liver, as well as enlargement of the head of the pancreas and the spleen with a normal gallbladder. An abdominal computed tomography scan showed pleural effusion, hepatosplenomegaly, enlargement of the pancreas, ascites and retroperitoneal lymphadenopathy. The cutaneous lesions biopsy revealed a leukocytoclastic vasculitis. The brain magnetic resonance imaging showed signs of diffuse atrophy. The diagnosis of SLE was established and steroid therapy was administered with a satisfactory outcome. Two months later she underwent a liver biopsy and a steatohepatitis was diagnosed. Afterwards, she was free of symptoms during a two-year period. 
Two episodes of abdominal pain with raised amylase and enlarged pancreas occurred thereafter, while she was on oral steroids and chloroquine, without other signs of SLE activity. At that time, serum levels of the IgG 4 subset of IgG were low.

In January, 2006, she had an admission due to neutropenia, fever and pneumonitis. She was on oral steroids and azathioprine (which were diminished and withdrawn, respectively). She had no abdominal symptoms at that time. Nevertheless, an abdominal US and a CT revealed pancreatic calcifications, a pseudocyst in the head of the pancreas and an irregular and dilated Wirsung duct (fig. 1). Antibiotics for respiratory infection were administered and finally, she could be discharged from hospital.

Thereafter, she was hospitalized 4 more times presenting with abdominal pain radiating to the back, vomiting and hyperamylasemia; she was treated with parenteral fluids, analgesics, prednisone and cyclosporin. Endoscopic ultrasonography (EUS) confirmed the findings of CP. Magnetic resonance cholangiopancreatography (MRCP) showed irregular and dilated Wirsung duct and three pseudocysts (the biggest one was associated with the tail of the pancreas) (fig. 2). The elastase fecal test and the blood glucose level were normal. Because of intolerance to oral feeding and abdominal pain, enteral nutrition by naso-jejunal tube was initiated. Afterwards, the abdominal pain worsened in the setting of fever and hematocrit fall. CT revealed two pseudocysts (head and body of the pancreas) and a third large pseudocyst located in the pelvis $(11 \mathrm{~cm}$ in diameter) (fig. 3$)$. Due to clinical deterioration an urgent external drainage of the pelvic pseudocyst was carried out; the amylase and lipase values in the fluid were 21,000 and $41,000 \mathrm{IU} / \mathrm{l}$, respectively, and the culture was negative for microorganisms. A total of $800 \mathrm{ml}$ of fluid was drained during a period of 12 days. The patient was managed with antibiotics (imipenem) and parenteral nutrition and the outcome was good.

Nowadays she is pain-free, on treatment with proton-pump inhibitors, oral pancreatic enzymes and antioxidants as well as low steroid doses and chloroquine. Her nutritional state is improving. The last abdominal US (December, 2006) showed signs of chronic pancreatitis, but it did not show pseudocysts or collections.

\section{Discussion}

The pancreatic involvement in the scenario of SLE is uncommon and the reported cases mention predominantly acute forms of pancreatic disease. The association between SLE and acute pancreatitis was first documented in 1939 by Reifenstein et al. and there are nearly 80 cases reported during the last 30 years in the English literature [1-4].

The etiology of lupus-associated pancreatitis remains unclear. Multiple mechanisms may be involved: autoimmune events, vasculitis, noninflammatory vasculopathy and antiphospholipid antibodies-related thrombosis.

The association between SLE and CP is extremely rare. Up to now, only six cases have been reported in the English literature [5-9].

We report a case of a young patient who was diagnosed of SLE at the age of 26 and presented several episodes of acute abdominal pain and hyperamylasemia (the first one simultaneously with the onset of SLE) and who developed an established CP after a twoyear period.

We believe CP could be attributed to SLE in this case. The patient denies alcohol and tobacco consumption. There is no evidence for nutritional deficiency, hypertriglyceridemia or chronic hypercalcemia. The family history does not suggest hereditary pancreatitis. Autoimmune pancreatitis seems unlikely in the present case considering the characteristic findings of this entity described by Okazaki [10] and the diagnostic criteria proposed by Chari et al. [11]; patients with autoimmune pancreatitis appear to respond promptly to oral steroid therapy and have high IgG 4 levels as well as a narrowed main pancreatic duct. In our patient's case the onset and the outcome of the first episodes of acute abdominal pain were steroid doses-dependant, but then they 


\begin{tabular}{c|l|l|l}
$\begin{array}{r}\text { Case Reports in } \\
\text { Gastroenterology }\end{array}$ & $\begin{array}{l}\text { Case Rep Gastroenterol 2008;2:6-10 } \\
\text { D0I: 10.1159/000112861 }\end{array}$ & Published online: January 10, 2008 & $\begin{array}{l}\text { O 2008 S. Karger AG, Basel } \\
\text { ISSN 1662-0631 } \\
\text { www.karger.com/crg }\end{array}$ \\
\hline
\end{tabular}

became independent of the treatment (low or high doses of steroids - oral or parenteral administration - and immunosuppressive agents did not improve her condition).

The previously reported cases also occurred in women and they were aged 12-61 with an average of 41 years; our patient is below the average.

In the case of the youngest woman, reported by Penalva et al. [8], a SPINK-1 mutation was found; this mutation is consider as a disease modifier which lowers the threshold for initiating pancreatitis or worsens the severity of pancreatitis caused by other factors [12].

In most reported cases, chronic pancreatitis was diagnosed more than 7 years after the onset of SLE. In the case reported by Swol-Ben et al. [9], symptoms of leukoencephalopathy and CP were the only clinical manifestations at the onset of SLE and the patient had not had a history of episodes of abdominal pain; in the rest of the cases (including ours) several episodes of abdominal pain and increased pancreatic enzymes preceded the development of CP.

In the case reported by Hortas et al. [6], two years elapsed between the first episode of pancreatitis and the appearance of findings of chronic disease; this situation is similar to the present case.

Our patient shares some findings with most of the cases published in the literature such as the presence of pseudocysts and/or calcifications in the imaging procedures and the absence of exocrine or endocrine pancreatic insufficiency $[7,8]$.

In summary, we report a patient who presented her first episode of abdominal pain and hyperamylasemia at the onset of SLE and whose anarchical evolution was remarkable. The rapid calcification development, the anatomical location of the abdominal collections and their complete resolution with the administered treatment are of notice.

Fig. 1. CT: Dilated and irregular Wirsung duct (black arrow) and pseudocyst in the head of the pancreas (arrowhead).

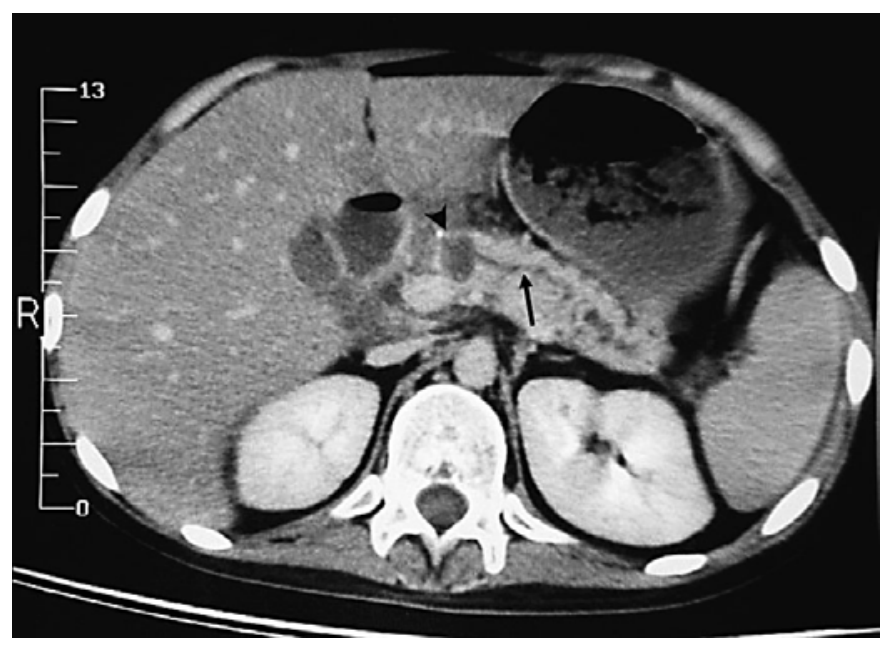


Fig. 2. MRCP: Irregular and dilated Wirsung duct (black arrow), pseudocyst in the head of the pancreas (arrowhead) and large pseudocyst associated with the tail of the gland (white arrow).

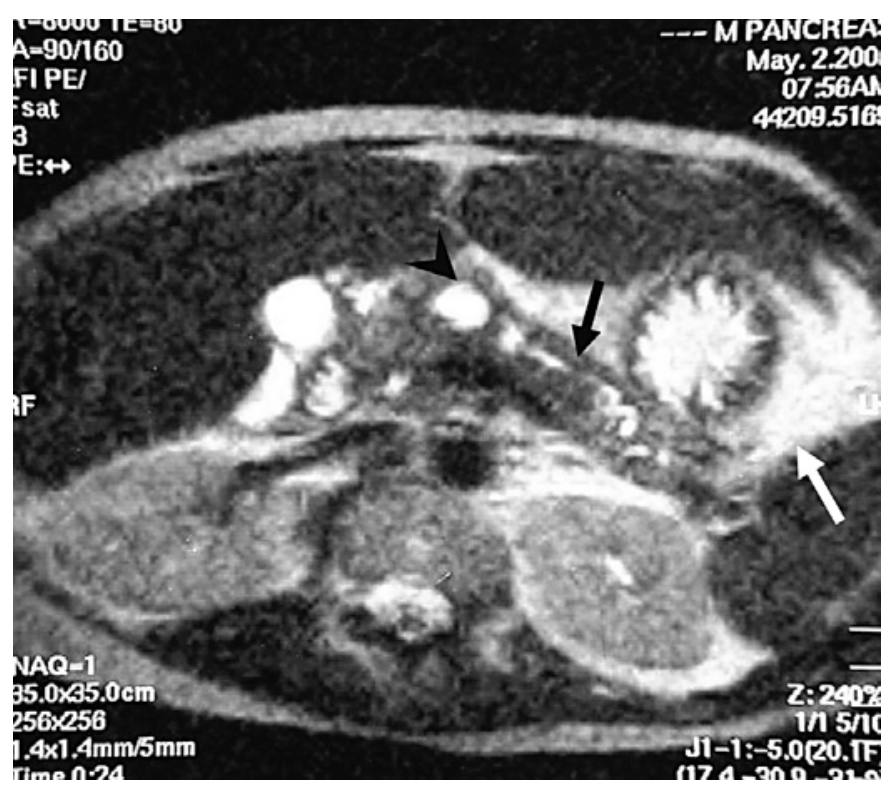

Fig. 3. CT: Large pelvic pseudocyst (black arrow).

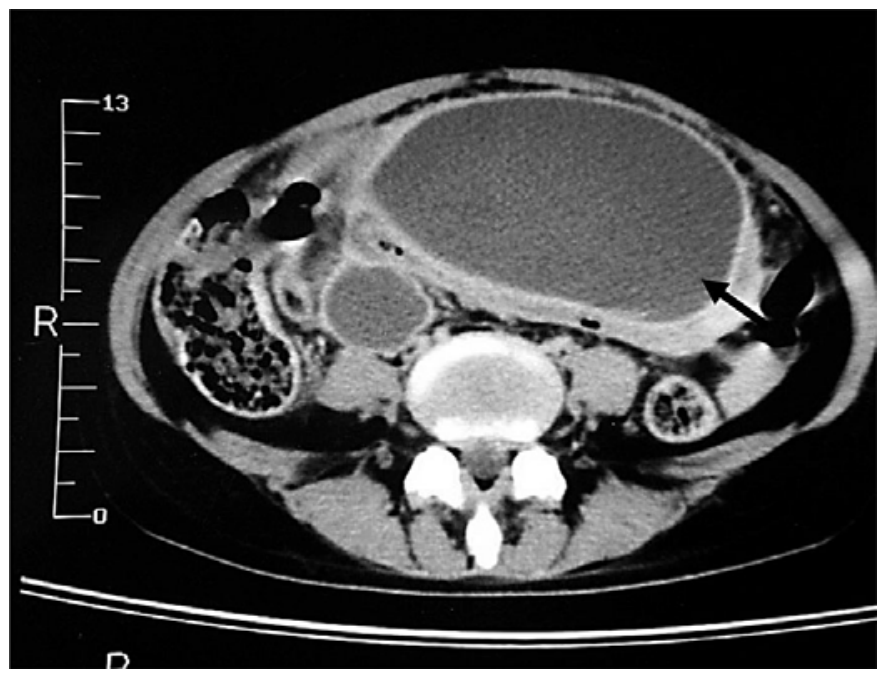




\section{References}

1 Derk C, De Horatius R: Systemic lupus erythematosus and acute pancreatitis: A case series. Clin Rheumatol 2004;23:147-151.

2 Duncan H, Achara G: A rare initial manifestation of systemic lupus erythematosus-acute pancreatitis: Case report and review of the literature. J Am Board Fam Pract 2003;16:334-338.

3 Nesher G, Breuer G, Temprano K, et al: Lupus associated pancreatitis. Semin Artritis Rheum 2006;35:260-267.

-4 Wang F, Wang NS, Zhao BH, Tang LQ: Acute pancreatitis as an initial symptom of systemic lupus erythematosus: A case report and review of the literature. World J Gastroenterol 2005;11:4766-4768.

5 Borum M, Steinberg W, Steer M, et al: Chronic pancreatitis: A complication of systemic lupus erythematosus. Gastroenterology 1993;104:613-615.

6 Hortas C, de Las Heras G, Lopez-Arias M, et al: Chronic calcifying pancreatitis in rheumatic diseases. Ann Rheum Dis 1995;54:77-78.

7 Izzedine $\mathrm{H}$, Caramella C, Ratziu V, et al: Chronic calcifying pancreatitis and systemic lupus erythematosus. Pancreas 2005;31:289-290.

$\checkmark 8$ Penalva J, Martinez J, Pascual E, et al: Chronic pancreatitis associated with systemic lupus erythematosus in a young girl. Pancreas 2003;27:275-277.

9 Swol-Ben J, Bruns C, Müller-Ladner U, et al: Leukoencephalopathy and chronic pancreatitis as concomitant manifestations of systemic lupus erythematosus related to anticardiolipin antibodies. Rheumatol Int 2004;4:177-181.

10 Okazaki K, Chiva T: Autoimmune related pancreatitis. Gut 2002;51:1-4.

11 Chari S, Smyrk T, Levy M, et al: Diagnosis of autoimmune pancreatitis: The Mayo clinic experience. Clin Gastroenterol and Hepatol 2006;4:1010-1016.

$\checkmark 12$ Etemad B, Whitcomb D: Chronic pancreatitis: Diagnosis, classification, and new genetic developments. Gastroenterology 2001;120:682-707. 